\title{
Digital Single-Operator Cholangioscopy in the Management of Biliary Strictures - Our Initial Institutional Experience
}

\author{
Petko Karagyozov ${ }^{1}$, Irina Boeva ${ }^{1}$, Ivan Tishkov ${ }^{1}$, Violeta Mitova ${ }^{1}$, Kiril Draganov ${ }^{2}$
}

'Department of Interventional Gastroenterology, Acibadem City Clinic Tokuda Hospital, Sofia, Bulgaria

${ }^{2}$ Clinic of Liver, Biliary, Pancreatic and General Surgery, Acibadem City Clinic Tokuda Hospital, Sofia, Bulgaria

\section{ABSTRACT}

Background: Biliary strictures (BSs) often represent a diagnostic challenge when evaluated with conventional endoscopic techniques. Many recent studies have focused on the specificity, sensitivity and accuracy of digital single-operator cholangioscopy (DSOC) in cases of BSs. However, DSOC is not a standardized procedure and the institutional experience with DSOC when used in different settings can provide important information.

Methods: This is prospective study of consecutive patients undergoing DSOC from March 2016 until March 2018. The aims of the study were to evaluate the role of DSOC for: (a) adequate visualization de of the target lesion and collection of biopsy samples adequate for histological evaluation and/or (b) traversing the BS under DSOC guidance when this was not possible under fluoroscopy. in addition we investigated thepossible influence of previous stenting on the cholangioscopic image and histology.

Results: Data on 44 consecutive DSOCs on 40 patients (median age 64.53 years, 24 male, 16 female) with BSs were collected All the tissue specimens were adequate for histological evaluation. The sensitivity of the cholangioscopic image was 100\% (95\% Cl, 82.4\%-100\%) and the specificity is $88.9 \%(95 \% \mathrm{Cl}, 51.8 \%-99.7 \%)$. The sensitivity of DSOC-guided biopsies was $62.5 \%(95 \% \mathrm{Cl}, 35.4 \%-84.8 \%$ ) and the specificity was $100 \%(95 \% \mathrm{Cl}, 59.0 \%$ $100 \%)$. There was no influence of previous stenting on the visual aspect and on the histological result $(p<0.05)$. The procedure was successful in all the cases where DSOC was used for guide wire stricture cannulation. Adverse events occurred in $6.8 \%$.

Conclusion: DSOC is successful in most cases and has high diagnostic yield with relatively low rate of adverse events.

Key words: cholangioscopy, digital single-operator cholangioscopy, indeterminate biliary strictures, adverse events

\section{INTRODUCTION}

The vast majority of biliary strictures (BSs) are malignant but data from surgical literature suggest that $15-24 \%$ of patients undergoing surgical resection of a suspected bile duct carcinoma have benign etiology $(1,2)$. BSs are considered to be indeterminate when a diagnosis can't be established upon

\author{
Corresponding author: \\ Kiril Draganov, MD \\ Clinic of Liver, Biliary, Pancreatic and \\ General Surgery, \\ Acibadem City Clinic Tokuda Hospital \\ 51B, N,Vaptzarov street, 1407, Sofia \\ Bulgaria \\ Tel.: + 35924034400 \\ GSM: + 359884933266 \\ GSM: + 359898561974 \\ E-mail: kiril_draganov2000@hotmail.com
}


basic laboratory work-up, abdominal imaging and ERCP with standard biliary sampling with brush cytology $(2,3)$. Some strictures are tight, fibrotic or angulated and they represent a serious challenge to endoscopists since the passage of a guide wire under fluoroscopic control might be impossible. The proper diagnosis of BSs in liver transplant patients can also be difficult when relying on "standard" imaging modalities.

Digital Single Operator Cholangioscopy (DSOC) visualizes the stricture itself and the method can provide targeted biopsies. DSOC has become a routine procedure in many high-volume centers being an integral and effective part of the ERCP armamentarium $(2,4-7)$. At the same time one must emphasize that additional interventions, such as percutaneous transhepatic cholangiography might be necessary to complete ERCP and DCS and to avoid surgery which is related with a high risk of severe specific morbidity (8). The aim of the study is to analyze the sensitivity, specificity and effectiveness of DCOS in our initial institutional series of patients with difficult for cannulation BSs.

\section{MATERIAL AND METHODS}

All cases of bile duct stenosis and/or obstructions who had undergone DSOC over a 2-year period (form March, 2016 until March, 2018) were included in a single-center, prospective, cohort study. Tumors of the head of the pancreas and the papilla of Vater were excluded. Thus a total number of 44 DSOCs were received by 40 patients with BSs.

Direct visual characteristics of strictures, histology of the specimen, specificity of endoscopic treatment and registered adverse events were analyzed. All the patients had previous abdominal imaging modalities ultrasound, CT-scan and magnetic resonance cholangiopancreatography. Those data had been taken in consideration before the procedure.

All the DSOCs were performed under general anesthesia and an antibiotic prophylaxis was given routinely.

At least 3 biopsy samples were taken using specially designed forceps (SpyBite, Boston Scientific) in cases of indeterminate biliary strictures and after careful evaluation of the stricture itself.

The final diagnosis was confirmed by histology (definitely positive for malignancy), surgery (in absolute surgical candidates) or clinical follow-up for at least 6 months (patients refusing surgery with negative histology for malignancy but malignant visual characteristics).

Descriptive statistical analyses were used and the results were presented as mean (range) and frequency (\%). DSOC and DSOC-guided biopsy were assessed by calculation of the diagnostic sensitivity and specificity and their exact confidence intervals based on the binominal distribution. Calculations were carried out by MS Excel 2016 as well as using available web-resource (9). Mann-Whitney Test and Kolmogorov-Smirnov Test were used to determine the relationships between previous stenting and visual diagnosis and previous stenting and histology and to determine the correlation between the cholangioscopic diagnosis and final diagnosis. A "p value" $<0.05$ was considered statistically significant. SPSS version 14.0 was used for the purpose of statistical analysis.

\section{RESULTS}

The male/female ratio 1.5/1 - 24 men and 16 women of a median age of 64.53 years (range $28-87$ years).

Thirty two of all 44 DSOCs (72.7\%) were diagnostic and 6 of them (13.6\%) were therapeutic. Two procedures (4.5\%) aimed both diagnosis and treatment and 4 DSOCs (9.1\%) were performed as follow-up examinations.

The procedures were successful and we obtained satisfactory views of the ductal lumen and the strictures in all the cases (figs. 1, 2).

Six patients from the whole series (15.0\%) had received liver transplantation in the past. They presented no diagnostic dilemma since a stricture in the anastomotic site (fig. 3). That's why those cases were excluded from the following analyses concerning the essence of the pathological process.

Cholangioscopic image suspected malignancy in $73.5 \%$ (25/34) and $26.5 \%$ (9/34) had benign visual signs. Twenty patients $(58.8 \%)$ had at least one biliary stent placed before referring for SOC. More than half of the oncological cases $(13 / 25,52.0 \%)$ had received stent placement in the bile ducts for at least 30 days before the scheduled SOC. DCS-guided biopsies were taken from 27 strictures (79.4\%) - 21/25 considered malignant and $6 / 9$ benign. The tissue specimens were sufficient and adequate for histologic evaluation in all the 27 patients (100\%).

Pathological report confirmed benign BSs in 6/6 cases that had been describes as benign at DCS. Follow-up DSOCs were performed in $2 / 6$ patients in a 3-month and in a 6-month period and they found no progression of the stricture and the procedure reconfirmed benign histology.

Malignancy was definitively proved in $10 / 21$ (47.6\%). Pathological report was inconclusive in $4 / 21$ 


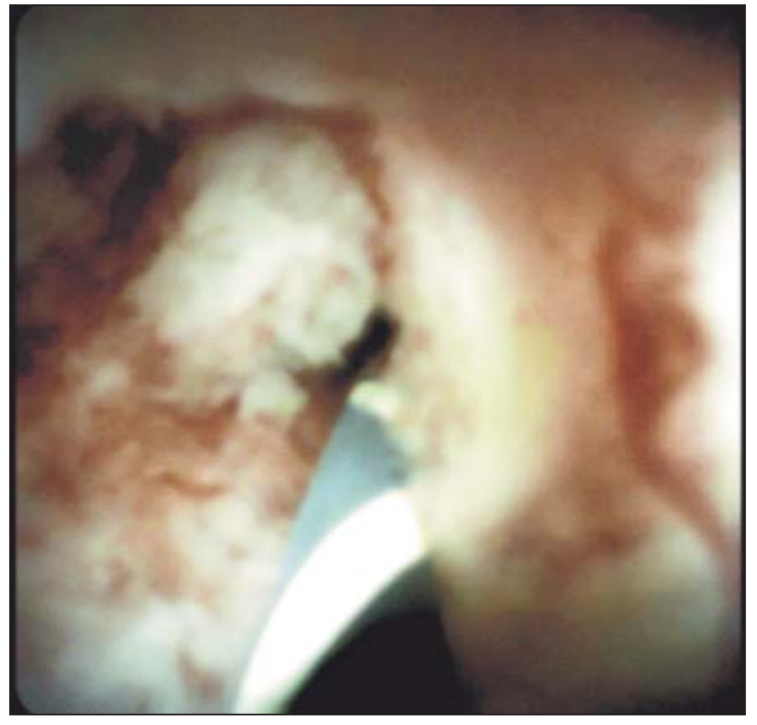

Figure 1 - Guidewire cannulation of the common bile duct under direct visual control

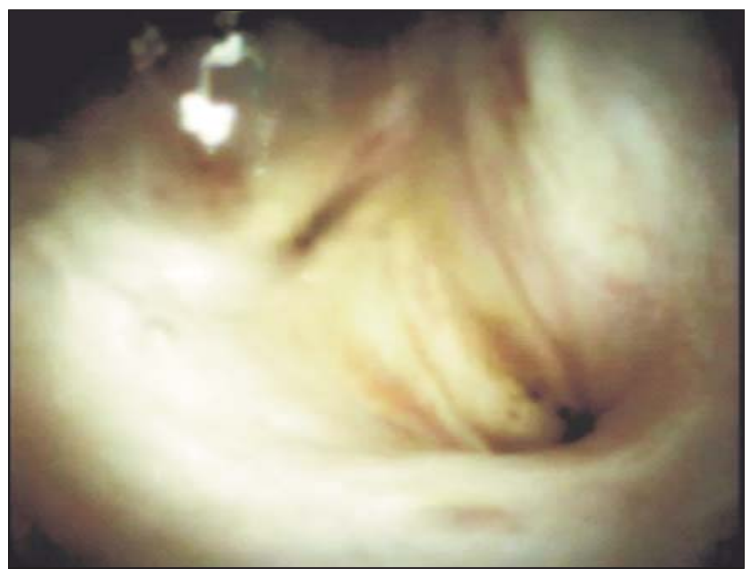

Figure 3 - Biliary anastomotic stricture after liver transplantation

patients (19.0\%) with malignant visual aspect of the strictures (fig. 4). However the malignant essence of the stricture was recognized at surgery in 2 from those 4 "inconclusive" patients - an early T-stage carcinomas were found at exploration and were resected (fig. 5). Pathologists described and reported benign characteristics in 7/21(33.3\%) but surgical resection confirmed cholangiocarcinoma in one of them.

Eight patients with malignant visual signs of the strictures and negative $(6 / 8)$ or inconclusive $(2 / 8)$ histology were followed-up clinical because of serious concomitant diseases and/or advanced age ("poor surgical candidates" $-7 / 8)$ and definite refusal of surgery (1/8).

Two patients with inconclusive and 1 with negative histology were followed-up and all of them showed progression of the malignant disease in a 3- to 6-month

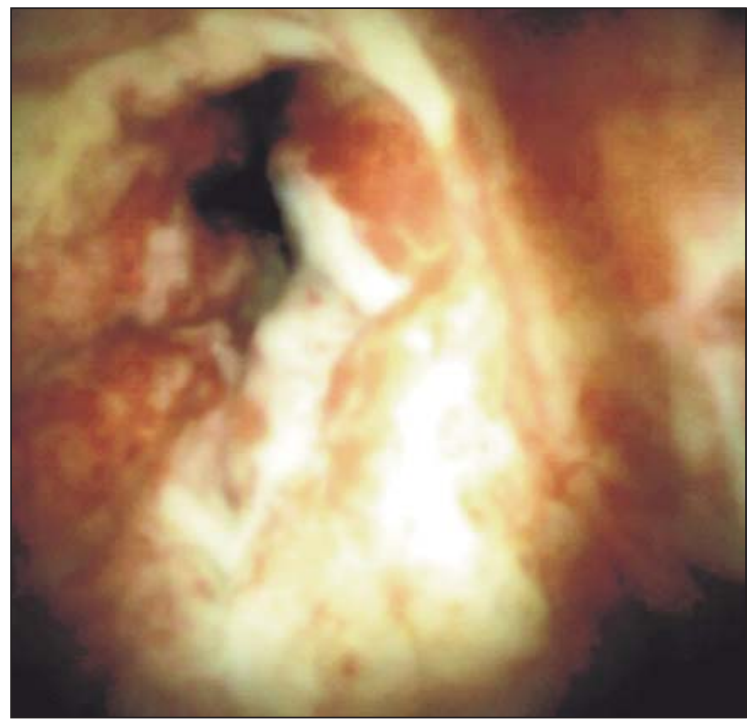

Figure 2 - Malignant biliary stricture - common bile duct cancer

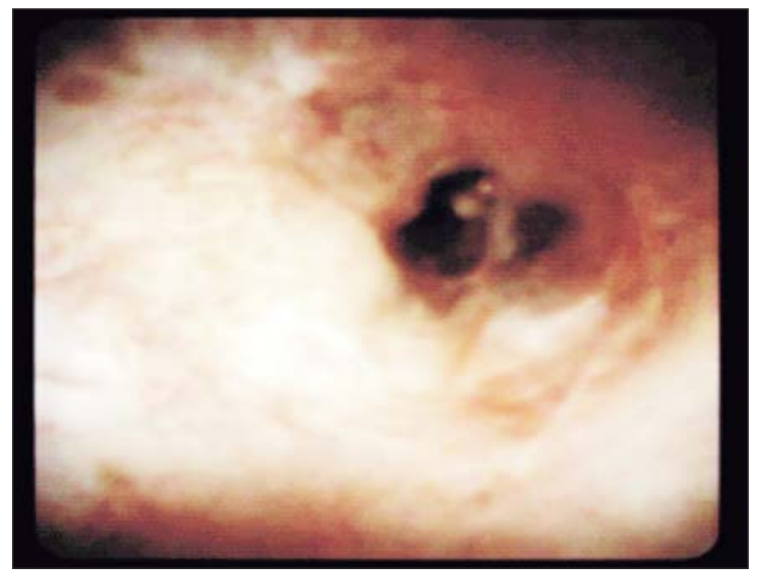

Figure 4 - Biliary stricture with visible "tumor vessels" but false negative histology

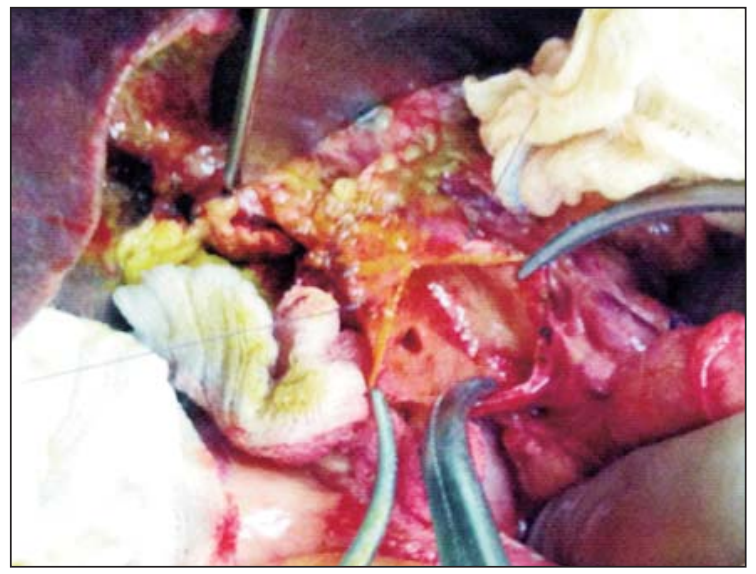

Figure 5 - Intraoperative finding - segmental common bile duct infiltrating cancer

(the orifice of the cystic duct is visualized) 
period which definitely confirmed the diagnosis.

We excluded 7 patients from the study because of certain endoscopic malignant signs but benign histology (3/7), lost for follow-up (1/7) and short (< 6 months) follow-up period (3/7).

A 45-year old female patient demonstrated cholangioscopic criteria that suggested malignancy but histology was inconclusive and the patient definitively refused surgery. She was followed-up for the next 2 years by performing DSOCs on the 3rd and every 6th month after the initial procedure. No progression of the changes was found and both specimen had negative histology. Benign biliary stricture was the final diagnosis.

Cancer was definitely confirmed in 95.0\% - 19/20 cases undergoing diagnostic DSOC with visual impression of malignancy and biopsies. The diagnosis changed from malignant to benign in $5.0 \%(1 / 20)$. Finally malignancy was diagnosed in all the cases with inconclusive histology but malignant visual aspect. All the strictures suspected as benign (6/6) were confirmed histologically.

The sensitivity of endoscopic diagnosis in this study was $100 \%$ (95\% Cl, $82.4 \%-100 \%)$ and the specificity $88.9 \%(95 \% \mathrm{Cl}, 51.8 \%-99.7 \%)$. The sensitivity and specificity of DSOC-guided biopsies were $62.5 \%(95 \% \mathrm{Cl}$, $35.4 \%-84.8 \%)$ and $100 \%(95 \% \mathrm{Cl}, 59.0 \%-100 \%)$ respectively. The sensitivity of the DSOC visual appearance for malignancies was $100 \%$ (95\% Cl, $82.4 \%-100 \%)$. The sensitivity of DSOC-guided biopsies confirming malignancy was $62.5 \%$ (95\% Cl, 35.4\%-84.8\%).

The statistical analysis showed a significant correlation between the cholnagioscopic diagnosis and the final diagnosis $(p<0.05)$. Any previous stenting didn't influence the strictures' visual aspects and nor the histologic result ( $p>0.05)$.

DSOCs were successful for selective guidewire cannulation after failure of the standard ERC-technique despite the use of different guidewires and occlusion balloons in 5 cases ( 4 malignant and 1 benign BS). One or two guidewires were successfully inserted through the strictures under direct vision in $100 \%(5 / 5)$.

DSOCs were performed in 6 patients with biliary complications after OLT. Four of the patients had complex strictures of the biliary anastomosis and attempts for a guidewire cannulation were not successful under fluoroscopic guidance. Anastomoses were visualized in all those 4 cases and in 3/4 the guidewire was successfully inserted through the stricture, facilitating further management. A case of anastomotic dehiscence associated with a stricture was successfully visualized but could not be bridged endoscopically and was referred for surgery. Two diagnostic DSOCs were performed. A case of ischemic hilar stricture with biliary casts obstructing the common hepatic and common bile ducts was managed successfully by irrigation and balloon extraction. The second case was suspected for an anastomotic stricture. DSOC demonstrated ischemiclike hilar stricture associated with intrahepatic strictures detected by magnetic resonance cholangiography. That patient was referred for re-transplantation.

DSOC-related adverse events rate was 6.8\% (3 patients). Complications included a case of cholangitis (prolonged hospital stay and a 5-day antibiotic course), acute cholecystitis (laparoscopic cholecystectomy performed) and a distal CBD perforation (recognized during the procedure and successfully treated with SEMS). No cases of acute pancreatitis or massive bleeding were registered as well as no complications affected the liver transplant group.

\section{DISCUSSION}

DSOC is effective in the management of BSs $(2,4)$. Visual signs of a stricture are quite reliable to confirm suspected malignancies and biopsy samples taken under direct visual control are conclusive in most cases (2). Typical cholangioscopic features indicating malignancy are irregularly dilated and tortuous vessels, easy oozing, irregular surface and variable degrees of an exophytic mass protruding into the lumen while smooth surface mucosa without neovascularization and lower homogenous papillo-granular mucosa without primary mass suggests benign lesion $(2,4,5,7,10)$.

Recent studies on DSOC demonstrated sensitivity and specificity of visual findings predicting malignancy of $88.9-100 \%$ and $76.0-97.7 \%$, respectively, a positive predictive value of $96.0 \%$ and a negative predictive value of $92.9 \%(2,4)$.

Reported sensitivity for DSOC-guided biopsy vary in a wide range in different series $(62.5-92.0 \%)$ while specificity if very high $(90.0-100 \%)(2,4)$. The unsatisfactory level of histology sensitivity in our study (62.5\%) is probably related to the absence of an access to on-site histology evaluation which could considerably improve this indicator. Published data suggest that the overall sensitivity of rapid on-site evaluation (ROSE) is $93.3 \%$ and specificity is $93.7 \%$ (11). Compared to biliary brush cytology and transpapillary biopsy, cholangioscopy guided biopsy demonstrated significantly higher sensitivity in this study. According published 2015 systematic reviews and meta-analyses the sensitivity of brushing is $45 \%$ and the sensitivity of intraductal biopsy is up to $48.1 \%$ (12).

Post-DSOC adverse events rate varies also a lot from $7 \%$ to $25.4(4,10,13)$. 


\section{CONCLUSION}

According to the results from our study the diagnosis of BSs often remains challenging despite the advances of the endoscopic technology.

DSOC provides sufficient visual information and increases the adequate biopsy rate in cases of malignant or benign BSs. The method can be successfully used in the endoscopic therapy for passage of a guidewire through strictures in cases of failure of the standard ERC-techniques. Follow-up DSOC is a suitable option to avoid unnecessary surgery in selected cases. SOC is a safe procedure and most of the adverse events can be treated conservatively.

\section{Acknowledgments}

The authors have no acknowledgments.

\section{Conflict of interests}

The authors declare no conflict of interests.

\section{REFERENCES}

1. Huszár 0 , Kokas B, Mátrai P, Hegyi P, Pétervári E, Vincze Á et al. Meta-Analysis of the Long Term Success Rate of Different Interventions in Benign Biliary Strictures. PloS. 2017;12(1): e0169618.

2. Urban O, Evinová E, Fojtík P, Lovecek M, Kliment M, Zoundjiekpon V, et al. Digital cholangioscopy: the diagnostic yield and impact on management of patients with biliary stricture. Scand $\mathrm{J}$
Gastroenterol. 2018;53(10-11):1364-1367.

3. Singh A, Gerlud A, Agarwal B. Biliary strictures: diagnostic considerations and approach. Gastroenterology Report. 2015;3(1): 22-31.

4. Lenze F, Bokemeyer A, Gross D, Nowacki T, Bettenworth D, Ullerich $\mathrm{H}$. Safety, diagnostic accuracy and therapeutic efficacy of digital single-operator cholangioscopy. United European Gastroenterol J. 2018;6(6):902-909.

5. Fukuda Y, Tsuyuguchi T, Sakai Y, Tsuchiya S, Saisyo H. Diagnostic utility of peroral cholangioscopy for various bile-duct lesions. Gastrointest Endosc. 2005;62(3):374-82.

6. Bhat YM, Kochman ML. Novel management of complex hilar biliary strictures with the Spyglass Direct Visualization System (with video). Gastrointestinal Endoscopy 2009; 69:1182-1184.

7. Navaneethan U, Hasan MK, Kommaraju K, Zhu X, Hebert-Magee $\mathrm{S}$, Hawes $\mathrm{RH}$, et al. Digital single-operator cholangiopancreatoscopy in the management of pancreatobiliary disorders: a multicenter clinical experience (with video). Gastrointest Endosc. 2016;84(4):649-55.

8. Martins Fernanda P, Ferrari Angelo P. Cholangioscopy-assisted guidewire placement in post-liver transplant anastomotic biliary stricture: efficient and potentially also cost-effective. Endoscopy. 2017;49:E283-E284

9. http://statpages.info/confint.html (last checked on 29 March 2018).

10. ASGE Technology Committee, Komanduri S, Thosani N, Abu Dayyeh BK, Aslanian HR, Enestvedt BK, et al. Cholangiopancreatoscopy. Gastrointest Endosc. 2016;84(2):209-21.

11. Hebert-Magee S., Hasan M, Navaneethan U et al. Improving the Diagnostic Yield of Single Operator Cholangioscopy (SOC)-Guided Biopsies of Indeterminate Biliary Strictures (IBS): Rapid on-Site Evaluation (Rose) to the Rescue? Gastrointestinal Endoscopy. 2016; 83:5S;AB: 134

12. Navaneethan U, Njei B, Lourdusamy V, Konjeti R, Vargo JJ, Parsi MA. Comparative effectiveness of biliary brush cytology and intraductal biopsy for detection of malignant biliary strictures: a systematic review and meta-analysis. Gastrointest Endosc. 2015;81(1): 168-76.

13. Korrapati P, Ciolino J, Wani S, Shah J, Watson R, Muthusamy VR, et al. The efficacy of peroral cholangioscopy for difficult bile duct stones and indeterminate strictures: a systematic review and meta-analysis. Endosc Int Open. 2016;4(3): E263-75. 